\title{
Michel PICHETTE
}

professeur associé à l'École des médias de l’Université du Québec à Montréal

et membre du Groupe de recherche sur les usages et la culture médiatique depuis 2005

\section{(Octobre 1968)}

\section{"Le syndicalisme \\ contre la révolution?”}

Un document produit en version numérique par Jean-Marie Tremblay, bénévole, professeur de sociologie au Cégep de Chicoutimi

Courriel: jean-marie tremblay@uqac.ca

Site web pédagogique : http://www.uqac.ca/jmt-sociologue/

Dans le cadre de la collection: "Les classiques des sciences sociales"

Site web: http://classiques.uqac.ca/

Une collection développée en collaboration avec la Bibliothèque

Paul-Émile-Boulet de l'Université du Québec à Chicoutimi

Site web: http://bibliotheque.uqac.ca/ 
Cette édition électronique a été réalisée par Jean-Marie Tremblay, bénévole, professeur de sociologie au Cégep de Chicoutimi à partir de :

Michel Pichette

“Le syndicalisme contre la révolution ?” (octobre 1968)

Un article publié dans la revue Socialisme 69, Revue du socialisme international et québécois, no 19, octobre-décembre 1969, pp. 11-18.

\begin{tabular}{ll|} 
M Pichette, professeur associé à l'École des médias de \\
l'Université du Québec à Montréal et membre du Groupe de \\
recherche sur les usages et la culture médiatique depuis 2005, \\
nous a accordé le 2 mai 2006 son autorisation de diffuser élec- \\
troniquement cet article dans Les Classiques des sciences so- \\
ciales.
\end{tabular}

8f Courriel : michel.pichette@gmail.com

Polices de caractères utilisée :

Pour le texte: Times New Roman, 14 points.

Pour les citations : Times New Roman, 12 points.

Pour les notes de bas de page : Times New Roman, 12 points.

Édition électronique réalisée avec le traitement de textes Microsoft Word 2004 pour Macintosh.

Mise en page sur papier format : LETTRE (US letter), 8.5'’ x 11'’)

Édition numérique réalisée le 11 février 2007 à Chicoutimi, Ville de Saguenay, province de Québec, Canada.

\section{Fait avec}




\section{Michel Pichette (octobre 1968)}

\section{“Le syndicalisme contre la révolution ?”}

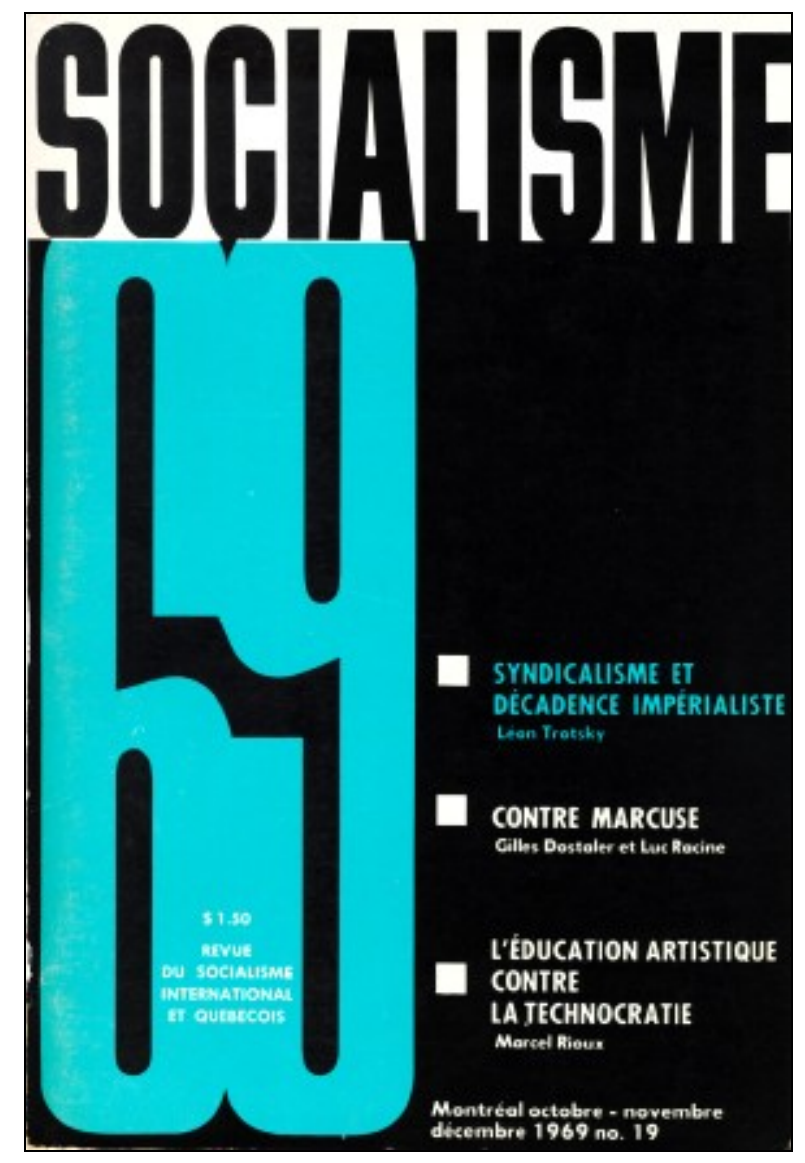

Un article publié dans la revue Socialisme 69, Revue du socialisme international et québécois, no 19, octobre-décembre 1969, pp. 11-18. 


\section{Table des matières}

\section{Introduction}

I. Syndicat - Rapports de production - Idéologie

II. -Collaboration syndicalisme - capitalisme 
Michel Pichette

professeur associé à l’École des médias de l’Université du Québec à Montréal membre du Groupe de recherche sur les usages et la culture médiatique

“Le syndicalisme contre la révolution ?”

Un article publié dans la revue Socialisme 69, Revue du socialisme international et québécois, no 19, octobre-décembre 1969, pp. 11-18.

"Dans sa lutte contre le pouvoir collectif des classes possédantes, le prolétariat ne peut agir comme classe qu'en se constituant en parti politique distinct... La coalition des forces ouvrières, déjà obtenue par la lutte économique, doit aussi servir de levier aux mains de cette classe contre le pouvoir politique." MARX ${ }^{1}$.

"Les conditions économiques avaient d'abord transformé la masse du pays en travailleurs. La domination du capital a créé à cette masse une situation commune, des intérêts communs. Ainsi, cette masse est déjà une classe vis-à-vis du capital, mais pas encore pour elle-même. Dans la lutte (...) cette masse se réunit, elle se constitue en classe pour soi. Les intérêts qu'elle défend deviennent des intérêts de classe. Mais la lutte de classes est une lutte politique." MARX ${ }^{2}$.

\section{Introduction}

$\underline{\text { Retour à la table des matières }}$

Au moment où le problème de l'organisation d'un mouvement révolutionnaire, dans une société capitaliste qui a survécue aux premières secousses de la révolution prolétarienne, pose l'urgence d'une théorie et d'une stratégie remises à jour en face de conditions objectives nouvelles, il s'impose de détruire les illusions que véhicule l'une des

1 Article 7a des Statuts de la Première Internationale (1866).

2 Misère de la Philosophie. [Texte disponible dans Les Classiques des sciences sociales. JMT.] 
formes d'organisation "non-politique" de la classe ouvrière, le syndicalisme.

Les conditions de vie nouvelle créées par le capitalisme monopoliste et l'idéologie de consommation qui lui est connexe véhiculent l'illusion de la participation et de la démocratie. La société industrielle capitaliste et le progrès technologique renforcent tout un processus de domination et de coordination qui à son tour dirige le progrès et créer des formes de vie et de pouvoir qui réconcilient avec le système les forces fondamentalement, opposantes, et de ce fait, rendent vaines toute protestation au nom de perspectives historiques, au nom d'une libération révolutionnaire des forces productives. Nous sommes conviés à vivre dans l'“opulent society”, sans que soient requis de notre part d'autres efforts que ceux de consommer toujours plus. La société capitaliste contemporaine paraît ainsi capable d'empêcher tout changement, toute transformation qui établirait des institutions essentiellement différentes, un mode de production radicalement différent, des rapports sociaux nouveaux.

Le syndicalisme dont l'évolution a suivi et suit de près celle des rapports de production capitaliste, apparaît d'une façon manifeste comme l'un des mécanismes d'intégration de la classe ouvrière au système établi et comme l'un des véhicules importants de l'illusion démocratique et égalitaire. Instrument de collaboration de classe au niveau non seulement économique. mais aussi aux niveaux politique et idéologique. il empêche la manifestation de la lutte des classes.

"Les syndicats. écrivait MARX, agissent utilement comme centre de résistance aux empiètements du capital. Ils échouent en partie quand ils font un usage peu judicieux de leur puissance. Ils échouent entièrement quand ils se livrent à une simple guérilla contre les effets du système capitaliste, au lieu d'essayer dans le même temps de le changer, au lieu de se faire un levier de toutes leurs forces organisées, pour l'émancipation finale de la classe ouvrière, c'est-à-dire, pour abolir enfin le prolétariat."

Il n'existe pas de faits dans l'histoire du syndicalisme (et non pas de la classe ouvrière) qui permettent de fonder sur lui quelque espoir révolutionnaire. Si le syndicalisme a pu constituer une "force d'appoint à 
l'organisation politique révolutionnaire de la classe ouvrière" (LENINE) en certaines circonstances, ce n'est pas dans la nature même du syndicat qu'il faut en chercher les explications, mais dans l'utilisation que faisaient les travailleurs de leur syndicat à l'intérieur d'une conjoncture révolutionnaire. En somme, le type d'organisation économique (et politique) que constitue le syndicalisme, ne peut être "révolutionnaire" que dans la mesure où la classe ouvrière elle-même, guidée par un parti révolutionnaire, s'empare du syndicat et lui fixe des objectifs de lutte économique "révolutionnaire". Sans cela, le syndicat constitue une force conflictuelle mais capitaliste et n'est pas une force d'opposition réelle et anti-capitaliste.

Quand cette "prise en main" du syndicat ne s'est pas effectuée, jamais ce dernier n'a servi de force d'appoint réelle au mouvement révolutionnaire. A aucun moment il ne s'est opposé à la société et à son État pour détruire l'une et l'autre, mais uniquement dans le but d'y conquérir une place et de s'y installer. En 1917, par exemple, c'est à partir des soviets et non des syndicats que la révolution russe s'effectue. En Allemagne, en 1918, les syndicats sont les premiers à freiner un mouvement révolutionnaire issu du prolétariat. lis déploieront leurs énergies à contenir le mouvement dans les limites favorables à la bourgeoisie. En Espagne. en 1936. l'action concertée de la CNT-UGT contre les comités ouvriers favorise l'installation à vie de Franco. A Cuba c'est aussi sans les syndicats et contre eux que Castro parvient à renverser le régime Batista. Enfin, lorsque surgissait en France, au mois de mai 1968 un mouvement de grèves généralisées (déclenché à la "base", sans le feu vert des dirigeants syndicaux), le premier geste de la CGT communiste et, avec elle, des centrales » rivales F.O. et C.F.D.T., fut de porter sur une base de négociation avec le patronat et l'État et de traduire en des termes de revendications "apolitiques" auprès du pouvoir ce qui avait toutes les caractéristiques d'une remise en cause non seulement du pouvoir gaulliste mais aussi de la structure même du pouvoir. L'attitude des centrales syndicales françaises (dont l'une, la CGT, est constamment identifiée dans les typologies --classiques à un syndicat révolutionnaire...) n'a pas peu contribué à l'avortement du mouvement de la classe ouvrière.

Il importe donc d'élaborer une stratégie de la révolution qui ne prenne pas pour acquis que le syndicalisme constitue une force d'ap- 
point au mouvement révolutionnaire. Les syndicats ne peuvent constituer cette "force" qu'à certaines, conditions dont la plus importante est qu'ils doivent être orientés révolutionnairement par les travailleurs eux-mêmes, ce qui implique une totale indépendance des, syndicats vis-à-vis l'État et la structure des rapports de production capitalistes, et l'instauration d'une démocratie réelle par la base.

Nous voulons dans ce texte faire apparaître, quelques-unes des raisons qui nous justifient de ne pas fonder "à tout prix" d'“espoirs” révolutionnaires dans les syndicats. Plus. nous voulons montrer en quoi il n'est pas dans la nature concrète du syndicat d'être une "force" au service des intérêts de classe de la classe ouvrière. Il apparaît plutôt comme un "ennemi" de classe et ce, malgré tous les avantages économiques immédiats dont il peut être responsable. Nous disons bien : le syndicat est une forme d'organisation capitaliste de la classe ouvrière. Nous ne disons pas pour autant qu'il ne peut pas. compte tenu de certaines conditions, constituer une force d'appoint à la lutte révolutionnaire.

L'étude des divers mécanismes d'étouffement de la conscience de classe et de l'illusoire démocratie de participation dont le syndicalisme se fait aujourd'hui l'un des principaux instruments et l'un des propagateurs se pose avec urgence, si nous voulons non seulement définir les termes de la lutte, mais aussi savoir où se situent les "forces d'appoint" possibles et impossibles d'un mouvement révolutionnaire. Puisqu'on ne change pas les structures d'une société en ne changeant que ses dirigeants, comment se fait-il que malgré leur "bonne volonté" les syndicalistes "de gauche" n'arrivent pas plus aujourd'hui qu'hier à faire du syndicat cet instrument d'appoint à la lutte anti-capitaliste dont ils rêvent ? Serait-ce que le syndicat est un mode d'organisation fondamentalement capitaliste ou encore, récupéré à son avantage par le pouvoir dominant?

Dans une société où la structure des rapports de production continue à exploiter les travailleurs, à confiner ces derniers à un agir par délégation de pouvoir et où le pouvoir dominant s'appuie sur J'existence de médiateurs qui limitent et normalisent l'action pour empêcher la formation d'une conscience de classe révolutionnaire chez les tra- 
vailleurs, il s'impose de nommer et d'analyser ce qui dans cette société empêche l'affirmation de la conscience et de l'action révolutionnaire.

\section{Syndicat - Rapports de production - Idéologie}

$\underline{\text { Retour à la table des matières }}$

Fondamentalement le syndicat est un mode d'organisation des forces productives dans la structure des rapports de production capitaliste. Ceci dit, il faut préciser que toute étude du syndicalisme doit être celle d'une forme spécifique d'organisation de la classe ouvrière dans ses rapports avec l'histoire du développement des forces productives à l'intérieur de formations sociales déterminées et de leur rapport avec le mode de production économique dominant. Cette précision est d'autant plus importante qu'elle nous oblige à faire une nette distinction entre ce qui peut être une force révolutionnaire et ce qui non seulement ne peut pas l'être mais ne l'est pas : les forcés productives peuvent se constituer en classe révolutionnaire contre les détenteurs de moyens de production et 'de la "plus-value", le syndicat ne peut pas.

Le syndicat peut se définir comme une association privée d'individus issus de certain secteurs productifs de l'économie et partant, formée d'une partie seulement de la "classe ouvrière". Par ailleurs, le syndicat est une organisation qui participe d'un système de "relations industrielles" par lequel il assure à ses "membres-cotisants" la satisfaction de leurs besoins immédiats de sécurité économique et sociale en même temps que leur insertion dans la structure de la société capitaliste. Cette définition "fonctionnaliste" nous laisse qu'une vision descriptive du syndicalisme : ce dernier est définie dans et par ses apparences immédiates. Il importe de la dépasser. Elle a toutefois pour intérêt de circonscrire l'un des cadres à l'intérieur duquel s'opère l'action syndicale : sa relation de dépendance vis-à-vis du pouvoir, sa reconnaissance implicite des lois de fonctionnement et de développement du mode de production capitaliste. 
Plus encore, le syndicat se trouve lui-même inséré dans la structure des rapports de production capitaliste. Ce n'est donc pas pour des raisons de "volonté", "d'intention", "d'orientation", etc. dont une organisation n'a que faire, qu'il est un facteur d'insertion (d'intégration de classe), mais parce qu'il est déjà partie composante dans des rapports de structures (politique, idéologique, économique) de domination de la classe ouvrière.

Situé à un point intermédiaire au niveau des rapports de production capitalistes, entre la classe ouvrière et les classes dominantes, le syndicalisme serait-il le résultat de ce que GRAMSCI appelle "un sacrifice d'ordre économico-corporatif consentie par les classes dominantes aux classes dominées, sans que pour autant une telle concession et un tel compromis n'altère l'essentiel de leur hégémonie" ? La place et le rôle qu'il a conquis dans la société capitaliste depuis le début du siècle et qu'il exerce avec force aujourd'hui, n'indiquerait-il pas que, malgré le caractère conflictuel de son action, il ne nuit en rien (au contraire) à des structures qui maintiennent l'opposition du capital et du travail ?

En ayant pour objectif de défendre les intérêts individuels et immédiats d'abord des artisans et des gens de métier et, ensuite, de la nouvelle main-d'oeuvre industrielle non spécialisée, le syndicalisme s'est structurellement modelé sur les lois de fonctionnement de l'économie capitaliste. Aujourd'hui particulièrement, dans les sociétés capitalistes (de l'ère post-industrielle. selon les thèmes éculées à la mode), l'action syndicale est strictement basée sur la revendication, et la combativité plus ou moins anarchique (refus de l'État, lois anti-trusts, etc...) des premières années a fait place à la volonté d'instaurer - par une collaboration avec le Pouvoir - des mécanismes socio-juridiques et politiques susceptibles d'engendrer une paix industrielle fondée sur l'harmonie des rapports entre le capital et le travail.

Il existe un lien étroit entre l'idéologie anarchiste et "révolutionnaire" des premières coalitions ouvrières et l'anarchie dans laquelle se développait la capitalisme libre-échangiste. C'est ainsi que la défense du petit contre le gros, de l'entreprise familiale contre le gros entrepreneur consiste souvent dans l'idéologie des premiers syndicats à rêver à ce que serait la société s'il n'y avait pas eu de révolution industrielle et à protéger, au détriment des consommateurs et des salariés, 
l'atelier vétuste, le commerce archaïque et l'exploitation agricole non rentable, à confondre des raisons humanitaires et révolutionnaires, la nécessité d'assurer des transitions supportables, aux moindres coûts humains, et le maintien de l'inefficacité économique et des privilèges établis.

Aussi, le trade-unionisme qui apparaît dès que s'instituèrent des mécanismes rationnels de négociation entre le monde du capital et le mon de du travail "organisé" eût-il de la facilité à s'accommoder d'un anti-capitalisme formel issu de résidus de l'idéologie traditionnelle dont l'anarcho-syndicalisme avait fait apparaître les plus profonds aspects.

Pour répondre à ses objectifs, le syndicalisme avait besoin de prendre des assises solides dans la nouvelle société et son alliance structurelle avec le capitalisme produit chez lui une idéologie axée sur le progrès, le développement et la "démocratie industrielle". L'anticapitalisme qui fait encore aujourd'hui le dénominateur commun du syndicalisme est finalement aussi vague qu'incertain. Il a plutôt la valeur d'une protestation globale contre l'irrationalité latente dans le développement économique capitaliste et d'une utopie plus qu'un programme révolutionnaire.

\section{II. - Collaboration syndicalisme - capitalisme}

$\underline{\text { Retour à la table des matières }}$

Les syndicats sont apparus en tant qu'organismes défensifs de la classe ouvrière face à des conditions de travail sous-humaines. lis se présentaient ainsi, sur le plan industriel et économique, comme le prolongement des anciennes confréries et corporations. Leurs aspirations se situent dès le premier regard, plus bas encore que celles du réformisme. Le réformisme, déployant des analyses idéologiques et économiques, prétend démontrer que, par le truchement de la démocratie capitaliste, il serait possible d'atteindre le socialisme par évolution légale et sans qu'il soit besoin d'actes révolutionnaires. Pour les syndicats il ne s'est agi ni d'évolution ni de révolution, encore moins de so- 
cialisme. Leur désir ne va pas au-delà de l'obtention pour le travailleur exploité de conditions moins intolérables et moins humiliantes, mais aussi plus rentables pour le capital. Tout en conservant une idéologie qui lui permet de se présenter comme la panacée aux problèmes du devenir historique, le syndicalisme a vite vu dans le parlementarisme et dans les exigences mêmes de l'accumulation du capital, l'heureux mécanisme d'une évolution certaine et harmonieuse de la classe ouvrière vers un paradis capitaliste de la consommation, de l'“opulence” et même de l'égalitarisme.

En se donnant pour objectif la défense des intérêts ouvriers dans le cadre de la société capitaliste, le syndicalisme se définit comme agent d'une collaboration de classe s'exprimant par la conciliation des intérêts opposés de ces classes. Si d'abord, il s'oppose au patronat, il ne tarde pas à se rendre compte que son rôle n'est pas situé sur le plan de la lutte. Il prend conscience de son importance en tant qu'intermédiaire entre les classes ennemies et, au lieu, d'animer le combat sur le terrain qui est le sien, ne. songe plus qu'au marchandage de l'armistice. Ce n'est pas la lutte qui justifie son existence mais sa valeur croit en proportion des résultats obtenus, à la fois aux yeux de patrons qui comprennent vite son importance et les services de médiation qu'il peut leur rendre et, aux yeux des travailleurs qui, peu à peu, s'en remettent aux seuls dirigeants du soin de trancher les litiges les opposant au patronat. La lutte de classes s'en trouve rejetée, leur autonomie disparaît, l'élan vers l'émancipation dégénère en accommodement dans le cadre capitaliste.

La pratique syndicale fait apparaître la contradiction entre une idéologie toute formelle empruntée à l'analyse marxiste de la lutte des classes et une attitude quotidienne inspirée de l'idéologie du capitalisme industriel, post-industriel, etc. Au plan politique, le syndicalisme collabore avec le pouvoir à l'élaboration des législations qui déterminent les mécanismes par lesquels il acheminera ses revendications, qui régiront les négociations avec le patronat et contrôleront les modes d'expression du mécontentement et des besoins des travailleurs. Au plan économique enfin, l'organisation syndicale se modèlera sur la concentration monopoliste capitaliste, au fur- et à mesure de son 
développement, et facilitera ainsi l'exercice de sa fonction de négociateur de la vente de la force de travail ${ }^{3}$.

HARBISON ${ }^{4}$ voit dans le "collective bargaining" un des moyens de l'intégration économique des travailleurs à la société américaine ; le sentiment qu'ils ont d'exercer un pouvoir sur leur environnement immédiat équivaudrait à' une sorte de participation à la société dans son ensemble (même si, selon cet auteur, il est peu probable que le "collective bargaining" ait un effet sur la redistribution du revenu national). Le "collective bargaining" n'a pas été imposé aux syndicats, mais sollicité par eux auprès du pouvoir qui ne demandait pas mieux 'd'encadrer et de régir par des lois fixes le domaine des "relations industrielles et du travail".

On peut se demander d'autre part si le fait de prévoir très minutieusement dans les contrats collectifs américains les conditions d'application de certaines décisions de la direction ne tend pas à sensibiliser les travailleurs plus à ses modalités qu'au fond même de la décision. Il est significatif par exemple que le "lay off" américain soit perçu comme une conséquence inéluctable des fluctuations du marché et de la production des commandes. Tout se passe comme si le fait que le "lay off" ait lieu selon les règles. "en toute équité" désamorçait les protestations, et comme si le cadre traditionnel de référence de l'action syndicale américaine, centrée sur la répartition des "rôles" au sein d'une

3 Nous pourrions faire une liste exhaustive ici des différents et nombreux mécanismes de collaboration : lois des relations patronales-ouvrières ; convention collective ; tribunaux du travail ; etc... Mais là n'est pas l'importance théorique de la question. En nous limitant à ce niveau des "faits observables" nous ne ferions que parler d'intégration institutionnelle, etc... au sens couramment employé en sociologie du travail. Par ailleurs, nous refusons de recourir au concept "d'action sociale" et de "sujet historique" pour expliquer ce qui n'est pas à expliquer, parce qu'inexistant au niveau de l'étude radicale du syndicat. Dans l'une ou l'autre perspective on arrive jamais a savoir exactement de quoi il parle. C'est pourquoi nous posons la. nécessité de situer l'étude dans le cadre d'une analyse de structures où, vidé de "ses orientations", de ses acteurs, etc... le syndicat apparaît dans la totalité de ses rapports avec les structures politique, idéologique et économique de la société globale.

4 Harbison, F., chapitre "Collective Bargaining and American Capitalism”, in Industrial Conflict, ouvrage collectif publié sous la direction de A. KORN HAUSER, R. DUBIN, A.M. ROSS, New-York, McGRAW Hill, 1954. 
quantité fluctuante, empêchait qu'une revendication de type "gestionnaire" - par exemple -, se fasse jour et que les mécanismes de la société globale soient regardés en face.

Si le syndicat assure la représentation et la défense des besoins qui naissent du travail et des conditions dans lesquelles il est accompli, et s'il apparaît capable de veiller à ce que les décisions économiques de l'État capitaliste tiennent compte de ces besoins, il reste inapte à dépasser un type d'action qui, axé sur le développement industriel, capitaliste, est orienté vers la résolution de ce que TOURAINE appelle "les problèmes modernes du contrôle social des modèles rationalisateurs" 5 !

Plutôt donc de chercher à résoudre la contradiction sociale fondamentale entre le capital et le travail salarié. le syndicalisme oeuvre à la résolution d'une contradiction tout à fait secondaire, interne aux intérêts de l'exploitation, celle de l'irrationalité du capitalisme, avec ses crises qui demandent un plan de production ordonné et une rigoureuse réglementation de la main-d'oeuvre, chômage compris. Ainsi coïncident les objectifs rationalisateurs du syndicalisme et ceux du grand capital qui réclame chaque jour plus de régulation économique, plus de concentration. En d'autres termes, ce que les syndicats perçoivent et veulent surmonter, ce sont les difficultés que le système rencontre sur sa route vers le monopole unique.

Plus la société capitaliste est vigoureuse, écrit le bien intentionné TOURAINE, moins alors le mouvement ouvrier la conteste, moins alors le syndicalisme est moderne en ce qu'il n'a pas à proposer un "modèle volontaire" de développement et de démocratie ${ }^{6}$. Cela veut dire que le syndicat s'identifie le plus aux objectifs de la structure sociale globale et qu'il agit à l'intérieur d'un rapport de collaboration (et de revendication) avec les organismes représentatifs et exécutifs de l'État capitaliste. Cependant le syndicalisme reste inapte à traduire les inconnus que sont les coûts sociaux "dérivés" de l'économie capitaliste en coûts sociaux "connaissables et mesurables", et de faire appa-

5 TOURAINE - Sociologie de l'action, chap. sur le mouvement ouvrier. Éditions du Seuil, Paris 1965.

6 TOURAINE, op. cit. 
raître les contradictions objectives inhérentes à l'application des décisions économiques ou d'un développement capitaliste, de manière à permettre à la collectivité de les résoudre réellement, au lieu de les éluder à la faveur de l'efficacité de la production.

Structurellement morcelée et brisée, la classe ouvrière se trouve condamnée à des luttes restreintes aux dimensions de l'entreprise ou de secteurs de production. Et, passant de la phase de libre compétition entre l'offre et la demande de force de travail (entre ouvriers et bourgeois) à la phase d'embrigadement de l'offre par la demande, c'est-àdire, des travailleurs par le capital monopolistique et étatique, les syndicats sont devenus les grands administrateurs de la vente de la maind'oeuvre à un coût de plus en plus régularisé (cf ; les luttes pour la parité des salaires) aux grands monopoles d'achat des forces productives.

Si l'émancipation des travailleurs doit être l'oeuvre des travailleurs eux-mêmes, ce qui constitue le postulat de toute action révolutionnaire authentique, il s'ensuit que le syndicalisme, étouffant le pouvoir créateur de la classe ouvrière, s'oppose à cette émancipation qui, avec lui, deviendrait l'oeuvre des dirigeants, s'ifs étaient capables de l'entreprendre ou même s'ils souhaitaient s'y consacrer.

Le contrat de travail qui fait l'objet de l'action revendicative et de la négociation des syndicats. une marque de fer pour la classe ouvrière. Collectif ou individuel, "libre" ou imposé. verbal ou écrit. le contrat de travail et le gribouillage légal de sa condition de classe esclave salariée par le capital. d'après les propres termes de MARX.

Effectivement, le contrat de travail, c'est-à-dire les conditions de location de la main-d'oeuvre. est élaboré par les syndicats (en l'occurrence par leurs services d'experts) après une entente négociée avec le patronat. Ainsi revient-il au syndicat la tâche d'encourager le servilisme productif par la promesse d'une meilleure paie, de réglementer par des lois les divers types de comportement des travailleurs au sein de l'entreprise et dans leurs rapports de classe au sein de la société globale.

Les lois régissant les négociations et les grèves, les mécanismes d'affiliation, l'organisation syndicale dans l'entreprise, etc., sont autant 
de réglementations approuvées par le syndicalisme qui favorisent un plus grand et plus efficace embrigadement de la classe ouvrière au service de la production capitaliste.

Bref, aux trois niveaux, économique, politique et idéologique, le syndicalisme porte la marque de son alliance structurelle avec le capitalisme. Non révolutionnaire, il constitue au contraire l'un des médiateurs les plus efficaces entre le capital et le travail. Se situant entre la conscience réelle et la conscience possible des travailleurs, il retarde la perception par eux du caractère illusoire des modèles de contrôle et de développement (de création et de démocratie), de l'illusoire humanité d'une société de consommation finalisée par l'accroissement rationalisée du capital et conséquemment, l'émergence de la conscience révolutionnaire.

Parlant pour les travailleurs et en leur nom, se présentant comme leur interlocuteur et leur répondant unique et officiel auprès du Pouvoir. il allie les travailleurs au système établi, sans qu'ils en aient conscience, et partant, confine au mutisme ceux qui les premiers ont a être entendus.

Aix-en-Provence, octobre 1968.

Fin du texte 